\title{
Zur Gliederung, Bildung und Altersstellung des Kalksteinschutts am Trauf der westlichen Schwäbischen Alb ${ }^{1}$ )
}

\author{
Von Dieter WeipPERT, Oberbrüden, Krs. Backnang \\ Mit 3 Abbildungen im Text
}

\begin{abstract}
$\mathrm{Z} u \mathrm{sammenf}$ as sung. Nach der Größe der Gesteinsstücke und dem Gehalt an Feinbestandteilen sind die Schuttmassen am Trauf der westlichen Alb drei verschiedenen Schuttarten zuzuordnen: dem „Schutt der Scherbenhalden“, dem „grobstückigen Kalkschutt“ und dem „feinsplitterigen Kalkschutt". Die beiden letzteren wurden während der Würmeiszeit gebildet und transportiert, während die Scherbenhalden hauptsächlich postglazial entstanden sind. Aus dem Profilaufbau, aus der Verbreitung und dem Tiefgang der vorhandenen Taschenböden ergibt sich, daß der „feinsplitterige Kalkschutt“ nur während ausgesprochener Kaltzeiten (Hochglaziale) und ste!lenweise (besonders am Fuße des Albtraufs) in situ aus ursprünglich grobstückigem Kalkschutt gebildet wurde.
\end{abstract}

$\mathrm{Summ}$ a ry. The steep slope to the NW of the Schwäbische Alb, the Albtrauf, is covered with thick layers consisting of limestone pebbles and cobbles. Because of the difference in grain size and in the percentage of loam, sand and silt mixed in, we can distinguish several different types of rubble: the pieces of the mountain screes, the "coarse rubble" an the "fine rubble". The "coarse rubble" and the "fine rubble" came into being during the Würm-glaciation. At the same time, they were transported by solifluction and spread all over the Albtrauf. The mountain screes are supposed to have been formed only in the postglacial area. The formation of the "fine rubble" did not appear before the time, where the ice reached its greatest extent. At some places, the "fine rubble files and by the extent and depth of the cryoturbated in-volutions found there.

Obwohl die Schwäbische Alb seit über 100 Jahren Gegenstand ausführlicher geologischer Forschung ist, sind deren jüngste Sedimente, die quartären Schuttmassen, nur wenig bekannt. Dies ist vor allem durch das Fehlen guter Aufschlüsse bedingt. Da der Bau der Bodensee-Fernwasserleitung zahlreiche neue Aufschlüsse und Profile lieferte, konnte nun der Versuch unternommen werden, die Schuttmassen am Albtrauf zu gliedern und zu datieren.

Herrn Prof. Dr. E. Seibold, Kiel und Herrn Dr. G. Einsele, Tübingen danke ich für Ihre Unterstützung und die Uberlassung der Grabenprofile, den Herren des Geologischen Landesamtes in Stuttgart und meinen Studienkollegen für vielerlei Hilfe und Anregung.

\section{Gliederung der Schuttmassen}

Da der Begriff "Schutt " im allgemeinen sehr weit gefaßt wird, muß hier zunächst eine Definition gegeben werden: Als Schutt bezeichne ich alle eckigen bis gerundeten Gesteinsbruchstücke über $2 \mathrm{~mm}$ Durchmesser, welche durch physikalische und chemische Verwitterung aus dem anstehenden Gestein entstanden sind und durch die Wirkung der Schwerkraft allein (Steinschlag) oder durch einen fließenden Bodenbrei transportiert wurden. Die Gesteinsbruchstücke mit 2 bis $0,02 \mathrm{~mm}$ Durchmesser (Kalksand) und die in die Schuttmassen eingemengten Mergel, Lehme und Tone werden zusammen als $\mathrm{Z}_{\mathrm{w}} \mathrm{i}$ $\mathrm{s} \mathrm{ch}$ e $\mathrm{n} \mathrm{m}$ i t t e 1 bezeichnet.

Schon bei einer Übersichtsbegehung fällt auf, daß die Schuttmassen am Albtrauf aus verschiedenen Schuttarten zusammengesetzt sind. Es lassen sich dabei drei Haupttypen unterscheiden:

1. Schutt der Scherbenhalden

2. Grobstïckiger Kalkschutt

3. Feinsplitteriger Kalkschutt.

1)Auszug aus einer 1959 angenommenen Dissertation (Mskr.) der math.-nat. Fakultät der Universität in Tübingen mit dẹm Titel: „Der Kalksteinschutt am Trauf der westlichen Schwäbischen Alb" 
1. Die $\mathrm{S}$ ch e r b e n h a lden (Schutthalden) werden aus eckigen Gesteinsbruchstücken aller Größen aufgebaut, welche im allgemeinen frei von einem Zwischenmittel sind. Sie tragen auch nur selten eine humose Bodenschicht, da deren Ausbildung durch die dauernde Anlieferung weiterer Gesteinsstücke verhindert wird. Diese Scherbenhalden findet man unter allen größeren Felsen des Albtraufs. Sie reichen je nach der Hangneigung und der Größe der Gesteinsstücke wenige Dekameter bis ca. $100 \mathrm{~m}$ die Hänge hinab.

2. Der grobstückige Kalkschutt bildet die Hauptmasse des Schutts am Albtrauf. Er setzt sich aus zentimeter- bis dezimetergroßen, eckigen Gesteinsbruchstücken zusammen. Das Zwischenmittel besteht hauptsächlich aus Kalksand und Mergeln, seltener aus Lehmen und Tonen. O'f ters enthält dieser grobstückige Kalkschutt dezimetermächtige Partien, deren Zwischenmittel ausgewaschen wurde.

3. Der feinsplitterige Kalkschutt tritt nur in bestimmten Horizonten zwischen dem grobstïckigen Kalkschutt auf und unterscheidet sich von diesem durch die geringere Größe der Gesteinsbruchstücke (meist nur zentimetergroß) und die etwas andere Sortierung des - überwiegend mergeligen - Zwischenmittels (vgl. Tab. 1). Teilweise

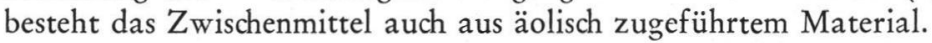

\begin{tabular}{lcccccc} 
& & $0,1-$ & $0,05-$ & $0,02-$ & \\
& $2-0,1$ & 0,05 & 0,01 & 0,002 & $<0,002 \mathrm{~mm} \phi$ \\
\cline { 2 - 6 } & 15 & 6 & 26 & 26 & 36 Gew. \% \\
grobstückiger Kalkschutt & 20 & 4 & 34 & 34 & 18
\end{tabular}

Tab. 1. Kornverteilung der Zwischenmittel bei grobstückigem und feinsplitterigem Kalkschutt

(Proben aus einem Profil bei Tuttlingen.)

Grobstückiger und feinsplitteriger Kalkschutt sind nur in natürlichen und künstlichen Anrissen erschlossen, da sie normalerweise von einem 30 bis $80 \mathrm{~cm}$ mächtigen Boden überdeckt werden. Beide Schuttarten bedecken die ganzen Hänge des Albtraufs und reichen häufig bis ins Albvorland und bis auf die Sohle der in die Albtafel eingeschnittenen Täler.

Da am Aufbau des Albtraufs Kalke der geschichteten Fazies und Massenkalke (Schwammkalke) beteiligt sind, läßt sich die oben gegebene Einteilung der Schuttmassen noch verfeinern: Bei den Scherbenhalden aus Massenkalk findet man Blöcke bis zur Größe eines kleinen Hauses, während die Kalke der geschichteten Fazies selten Stïcke mit mehr als $20 \mathrm{~cm}$ Kantenlänge liefern. Beim grobstückigen und feinsplitterigen Kalkschutt treten diese Größenunterschiede nicht besonders hervor, dagegen sind hier Unterschiede in der Form und Sortierung der Gesteinsbruchstücke aus verschiedenem Material vorhanden. Massenkalke lieferten hauptsächlich gut sortierte, feinere (2 bis $5 \mathrm{~cm}$ Durchmesser), eckige Gesteinsbruchstücke; Kalke der geschichteten Fazies dagegen schlecht sortierte, gröbere (5-20 cm Durchmesser) eckige Gesteinsbruchstücke. Die Gesteinsstücke aus Massenkalk sind meist würfelig bis quaderig geformt, die aus Kalken der geschichteten Fazies ent-
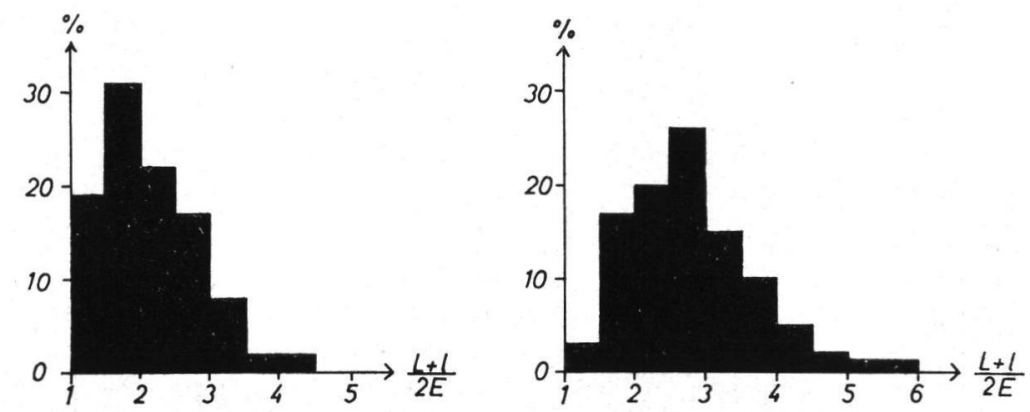

Abb. 1. Form der Gesteinsstücke (Abplattungsindex nach CAILLEux: $L=$ größte Länge, $1=$ größte Breite, $\mathrm{E}=$ größte Dicke). - a) Massenkalke des Weißjura $\beta, \mathrm{b})$ Kalke des geschichteten Weißjura $\beta$. 
standenen Stücke überwiegend scherbig, plattig. In Abb. 1 sind diese Formunterschiede mit Hilfe des Abplattungsindex' nach CaIlleux (1952) dargestellt.

Bei einem Vergleich verschiedener Schuttprofile muß besonders auf diese - materialbedingte - unterschiedliche Ausbildung der Gesteinsbruchstücke geachtet werden, da sonst bei einer klimatologischen Auswertung der Profile (vgl. S. 28 ff.) erhebliche Fehler entstehen. Auch sind deshalb die Begriffe "grobstückiger" und „feinsplitteriger" Kalkschutt nur relativ zu verstehen, da z. B. ein aus Massenkalk entstandener grobstïckiger Kalkschutt im Durchschnitt kleinere Gesteinsbruchstïcke enthalten kann als ein feinsplitteriger Kalkschutt, der aus Kalken der geschichteten Fazies gebildet worden ist.

\section{Zur Unterscheidung Schutt/Schotter}

Die Schuttmassen am Albtrauf wurden durch die Wirkung der Schwerkraft allein (Bildung von Schutthalden) oder durch einen fließenden Bodenbrei (Solifluktion) verfrachtet. Wo die Schuttmassen in die Nähe von Bächen und Flüssen gelangten oder direkt deren Täler erreichten, wurden sie von fließendem Wasser weitertransportiert. Während des fluviatilen Transports erfolgte eine Abrundung der vorher zumeist eckigen Gesteinsbruchstücke; gleichzeitig wurde die Zusammensetzung und Sortierung des Zwischenmittels verändert. Nach einem genügend weiten fluviatilen Transport lassen sich die entstandenen Schotter gut von Solifluktionsschutt unterscheiden. Dagegen wird diese Unterscheidung oft sehr schwierig, wenn es sich um Material handelt, das 1. nur über kurze Strecken fluviatil transportiert wurde oder 2. aus verwitterten Ablagerungen stammt.

1. Geringerfluviatiler Transport. Als Beispiel hierzu seien die Niederterrassen der Albtäler angeführt. Sie bestehen aus Material, welches über wenige km fluviatil transportiert und bald wieder abgelagert wurde. Diese Niederterrassen, die heute 3 bis $12 \mathrm{~m}$ über dem Bachniveau liegen, reichen weit in die Albtäler hinein und sind durch Kiesgruben gut erschlossen. Daß in den Albtälern eine Unterscheidung zwischen den Schottern der Niederterrassen und dem - nicht fluviatil transportierten - Schutt möglich ist, zeigen die Darstellungen der Rundungsverhältnisse (nach CAILleux 1952) bei frischen Schottern und frischem Solifluktionsschutt (Abb. $2 \mathrm{a}$ und $2 \mathrm{~b}$ ) und der Vergleich von Siebanalysen desselben Materials (Tab. 2): Die Niederterrassen enthalten stärker gerundete Gesteinsbruchstücke und einen geringeren Anteil an feinem Material als die - durch Solifluktion transportierten - Schuttmassen.

Sedimente der Niederterrassen

(5 Proben)

Solifluktionsschutt

(20 Proben)

\begin{tabular}{cccc}
$>10$ & $10-2$ & $2-0,1$ & $\langle 0,1 \mathrm{~mm} \phi$ \\
\hline 73 & 12 & 7 & $8 \mathrm{Gew} . \%$ \\
70 & 8 & 5 & 17
\end{tabular}

Tab. 2. Kornverteilung in den Sedimenten der Niederterrassen und im Solifluktionsschutt (Mittelwerte).

Allerdings handelt es sich bei den untersuchten Sedimenten der Niederterrassen um Material, das bereits über 1 bis $2 \mathrm{~km}$ fluviatil transportiert wurde. Bei einem geringeren Transportweg sind die oben angeführten Unterscheidungsmöglichkeiten meist nicht mehr brauchbar, und eine Trennung Schutt/Schotter ist oft unmöglich.

2. Verwitterte Ablagerungen. Wirkt die chemische Verwitterung längere Zeit auf die Schuttmassen ein (Ausbildung eines Bodenhorizonts), so werden die zuvor eckigen Gesteinsbruchstücke allmählich gerundet. In Abb. 2 (a bis c) sind die Ergebnisse von Rundungsmessungen an Niederterrassen-Schottern und an frischem und verwittertem Solifluktionsschutt einander gegenübergestellt. Die Zurundung der Gesteinsbruchstücke aus dem Verwitterungsbereich ist aus der Abbildung gut ersichtlich. Die vermessenen angewitterten Stücke stammen aus dem Boden, der sich während des Spätglazials und im 


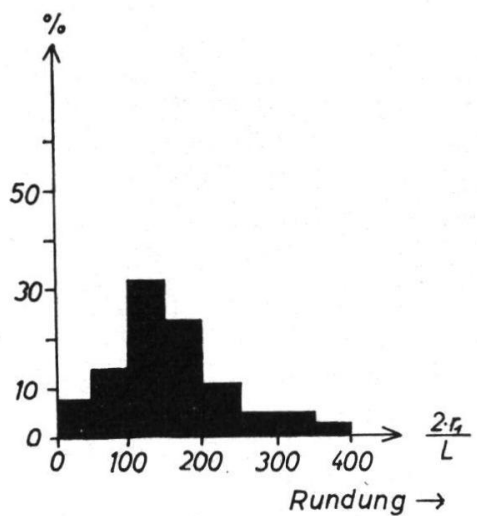

a) Frische Niederterrassen-Schotter

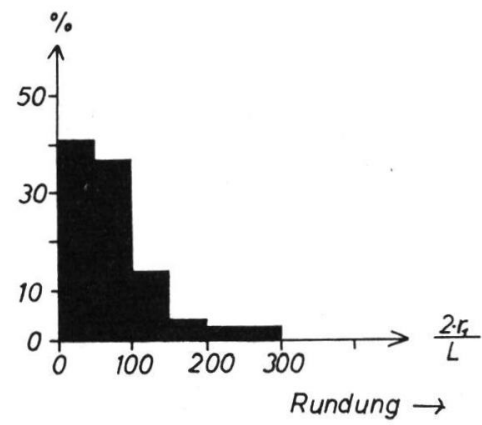

c) Angewitterter Schutt

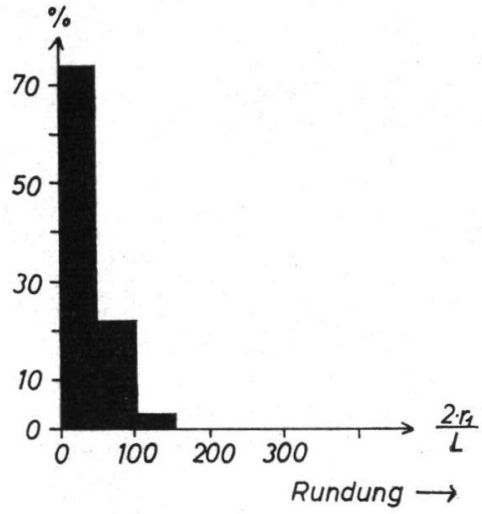

b) Frischer Schutt

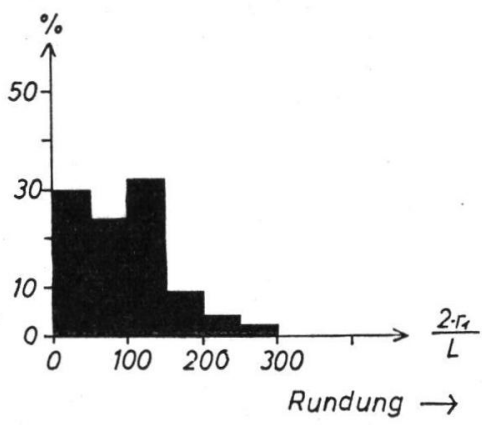

d) Mittelpleistozäne Ablagerungen

Abb. 2. Abrundung der Gesteinsstücke durch Transport und Verwitterung (Rundungsindex nach CaIlleux: $r_{1}=k$ leinster Krümmungsradius, $L=$ größte Länge).

Holozän entwickelte. Die Stücke waren also der chemischen Verwitterung nicht sehr lange ausgesetzt und sind deshalb im Rundungsdiagramm noch als Schutt zu erkennen.

Anders sind die Verhältnisse bei sehr alten Schuttlagen, die bereits während eines oder mehrerer Interglaziale und -stadiale der chemischen Verwitterung zugänglich waren. Dies soll an einer Probe aus vermutlich mittelpleistozänen Ablagerungen gezeigt werden: Nach dem Rundungsdiagramm (Abb. 2 d) könnte es sich bei diesen Ablagerungen sowohl um wenig verfrachtete Schotter als auch um stark verwitterten Schutt oder eine Mischung von beiden handeln. Auch versagt hier die Methode des Vergleichs von Siebanalysen, da die Ablagerungen in ihrer ganzen Mächtigkeit von der Verwitterung erfaßt wurden und die ursprünglichen Verhältnisse daher nicht mehr gegeben sind. Einen Hinweis auf die Natur dieser Ablagerungen gab aber der Zustand der Oberflächen der Gesteinsstücke. Während sich die Oberflächen bei stark verwittertem Schutt trotz einer dicken Verwitterungsrinde (mm-Beträge) beim Darüberstreichen mit dem Finger meist rauh anfühlen, zeigten die Stücke aus den untersuchten Ablagerungen eine dünne Verwitterungsrinde $(1 / 10 \mathrm{~mm}$-Beträge) und eine relativ glatte Oberfläche, wie sie Schottern eigen sind. Daß es sich bei dem bearbeiteten Material wahrscheinlich um wenig verfrachtete Schotter handelt, kann auch aus der Lage des damaligen Albtraufs geschlossen werden. Ebenso wie bei diesem Beispiel ist es bei alten Höhenschottern und fraglichen Schuttvorkommen im Albvorland nur an Hand der Lage, der Verbreitung und des Zustandes der Gesteinsstücke 
möglich, eine - vielleicht richtige - Entscheidung zu treffen. Messende Methoden versagen vorläufig noch.

\section{Bildung und Alter der Schuttmassen}

1. Di e Scherbenhalden, welche heute den obersten Teil der Hänge am Albtrauf bedecken, sind relativ jung und wohl alle erst während des Holozäns entstanden. $\mathrm{Da}$ aber keine brauchbaren Profile gefunden wurden, war eine genaue Datierung und eine Gliederung der Scherbenhalden nicht durchzufühuen.

2. Der grobstückige und feinsplitterige Kalkschutt dagegen war durch Kiesgruben und besonders durch den Graben der Bodensee-Fernwasserleitung gut erschlossen. Weil von dem gesamten Graben ein durchgehendes Profil gewonnen werden konnte (aufgenommen von Studenten des geologischen Instituts in Tübingen), stützen sich die folgenden Angaben vor allem auf dieses Grabenprofil.

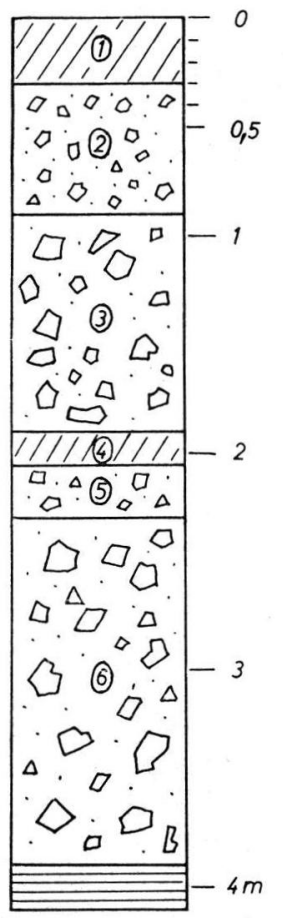

Abb. 3. Schuttprofil bei Spaichingen

(Graben der Bodensee-Fernwasserleitung).

$1,4=$ Verlehmungszonen

2,5 = feinsplitteriger Kalkschutt

3,6 = grobstückiger Kalkschutt

Unterlage (horizontal schraffiert): anstehender Dogger.

Die Lagerung des Schutts am Albtrauf ist besonders gut aus einem Profil bei Spaichingen zu ersehen (Abb. 3). Es enthält zwei, gleich ausgebildete Schuttfolgen: An der Basis liegt grobstückiger Kalkschutt (Schicht 6, bzw. 3). Darüber folgt eine Lage feinsplitterigen Kalkschutts (Schicht 5, bzw. 2), welche von einer Verlehmungszone (Schicht 4, bzw. 1) ïberdeckt wird. Daß diese Abfolgen nicht zufällig entstanden sind, beweist allein schon deren weite Verbreitung. Profile, welche zwcimal eine Abfolge grober Schutt/feiner Schutt/Verlehmungszone enthalten, wurden zwar nur in geringer Zahl ge.. funden (bei Spaichingen, bei Tuttlingen und am Dreifürstenstein bei Mössingen), doch läßt sich e in e derartige Abfolge, welche den Schichten 3 bis 1 in dem oben erwähnten Profil entspricht, in vielen Aufschlüssen am Fuße des Albtraufs nachweisen.

Die klimatologische Deutung der Schuttprofile wird durch die Arbeiten Woldstedts $(1956,1958)$ über den Temperaturverlauf und die Klimaschwankungen während einer Eiszeit ermöglicht (Tab. 3). 


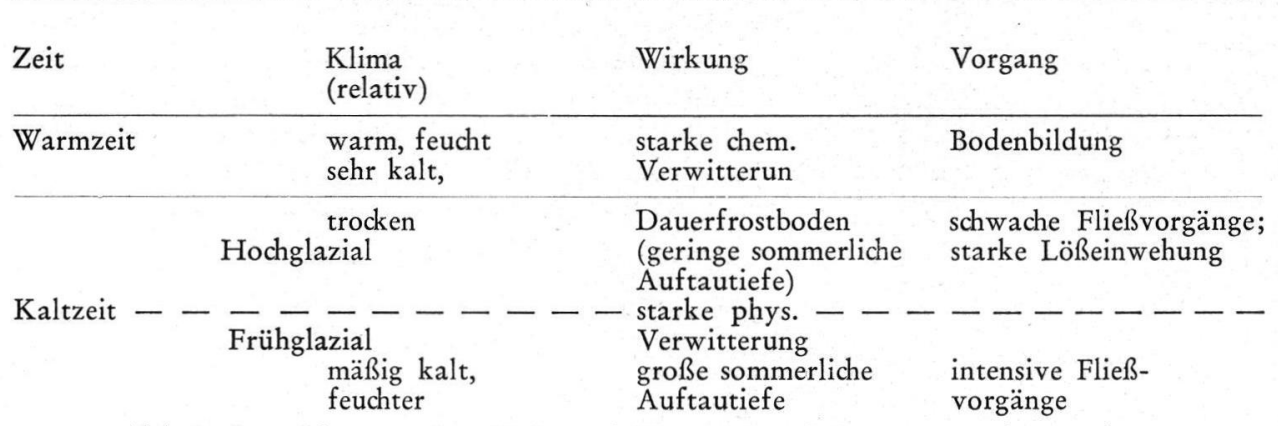

Tab. 3. Auswirkungen einer Kalt- und Warmzeit auf das immer eisfreie Gebiet (in Anlehnung an WoLDSTEDT)

Die Bildung der Abfolge grober Schutt/feiner Schutt ist folgendermaßen zu erklären: Die intensiven Fließvorgänge während eines Frühglazials lieferten die mächtigen Lagen aus grobstückigem Kalkschutt. Während des folgenden Hochglazials herrschte im Auftaubereich stärkere Frostsprengung, welche den feinsplitterigen Kalkschutt entstehen ließ. Der Schutt, welcher unterhalb der sommerlichen Auftautiefe lag, wurde nicht verändert.

Infolge der schwachen Bodenbewegungen während des letzten Hochglazials (Mittelwürm-Maximum; vgl. S. 30) blieben die entstandenen Dauerfrostformen (Taschenböden und Eiskeile) a m Fuße des A lbtraufs erhalten. Der Tiefgang dieser Formen - im Durchschnitt 1,5 m (Weippert 1960) - gibt uns ein ungefähres Maß für die sommerliche Auftautiefe während der Zeit ihrer Entstehung. Nun sind die - vielfach aufgefundenen - Taschenböden eindeutig epigenetischer Entstehung. Das bedeutet, daß die Schuttmassen am Fuße des Albtraufs während des letzten Hochglazials nicht mehr oder nur ganz unbedeutend bewegt wurden. Der feinsplitterige Kalkschutt (z. B. Schicht 2 auf Abb. 3) kann daher hier nur in situ aus ursprünglich grobstiickigem Schutt entstanden sein! Diese Ansicht wird gestützt durch die Tatsache, daß die Mächtigkeit des feinsplitterigen Kalkschutts häufig etwa gleich dem Tiefgang der Taschenböden ist. Deshalb gibt in diesem Falle auch die Mächtigkeit des feinsplitterigen Kalkschutts allein ein ungefähres Maß für die sommerliche Auftautiefe zur Zeit seiner Bildung.

Für die untere Lage feinsplitterigen Kalkschutts (z. B. Schicht 5 auf Abb. 3) ist eine derartige Entstehung in situ zwar nicht zu beweisen, aber doch anzunehmen. Die geringe Mächtigkeit dieser Schicht ist wohl durch Verwitterungs- und Abtragungsvorgänge während einer Warmzeit (Bildung der unteren Verlehmungszone) bedingt.

Es muß ausdrücklich betont werden, daß eine klimatologische Deutung dieser Art nur bei ungestörten Profilen möglich ist. Das sind am Albtrauf jene Profile, die weit vom Schuttliefergebiet entfernt sind und an flachen Hängen ( 2 bis $5^{\circ}$ Neigung) liegen. A n

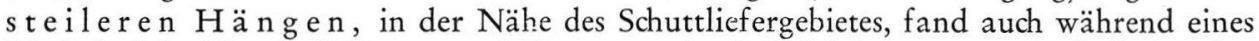
Hochglazials Schuttanlieferung und Transport durch Solifluktion statt. Deshalb wurden in den oberen Teilen der Hänge am Albtrauf auch keine Taschenböden gefunden, und eine Abfolge grobstückiger/feinsplitteriger Kalkschutt ist nur an wenigen, günstigen Stellen erhalten (vgl. BLeich \& Groschopf 1959). Der feinsplitterige Kalkschutt kann hier nicht in situ entstanden sein, und seine Mächtigkeit liefert folglich auch kein Maß für die sommerliche Auftautiefe während eines Hochglazials.

$\mathrm{D}$ as Alter der Schuttmassen. Bei den Untersuchungen zur Trennung von Schutt und Schottern ergab sich, daß die Schuttmassen am Albtrauf direkt mit den Niederterrassen verzahnt sind. Diese Verzahnung beweist ein würmeiszeitliches Alter des Schutts. Leider war keine Verbindung zwischen einer solchen Verzahnungsstelle und einem Profil, welches eine zweifache Abfolge grober Schutt/feiner Schutt/Verlehmungszone enthält, erschlossen. Das Alter der unteren Verlehmungszone ist deshalb nicht ein- 
deutig festzulegen. Da das Interstadial zu Beginn der Mittelwürmzeit, das „Göttweiger Interstadial“, wesentlich länger andauerte und mächtigere Bodenbildungen hervorgebracht hat als das jüngere „Paudorfer Interstadial“, werden die unteren Verlehmungszonen den Bildungen des "Göttweiger Interstadials" gleichgesetzt.

Abfolge und Alter der beschriebenen Vorgänge sind in dem folgenden Schema zusammengestellt:

\begin{tabular}{|c|c|c|}
\hline \multicolumn{2}{|c|}{ Alter } & mutmaßlicher Vorgang \\
\hline \multicolumn{2}{|l|}{ Postglazial } & Bildung der obersten Verlehmungszone (heutiger Bodenhorizont). \\
\hline \multicolumn{2}{|l|}{ Spätwürm } & $\begin{array}{l}\text { Geringer Schutt-Transport; Umlagerung der liegenden Schichten. } \\
\text { Beginn der Bodenbildung. }\end{array}$ \\
\hline & $\bar{M} \overline{\text { Maximum }}$ & 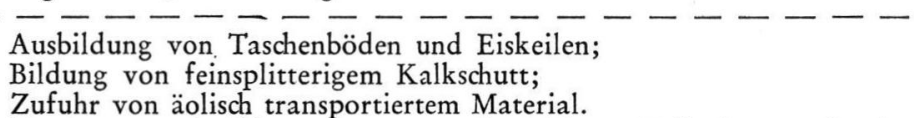 \\
\hline $\begin{array}{l}\text { Mittel- } \\
\text { würm }\end{array}$ & $\begin{array}{l}\text { Vorstoß- } \\
\text { phase } \\
\text { Interstadial }\end{array}$ & $\begin{array}{l}\text { Transport und Ablagerung von grobstückigem Kalkschutt; teilweise } \\
\text { Umlagerung der liegenden Schichten. } \\
\text { Bildung der unteren Verlehmungszone. }\end{array}$ \\
\hline Frühwürm & & $\begin{array}{l}\text { Gegen Ende der Frühwürmzeit Bildung von feinsplitterigem Kalk- } \\
\text { schutt. Intensive Fließvorgänge; starker Transport und Ablagerung } \\
\text { von grobstückigem Kalkschutt. }\end{array}$ \\
\hline
\end{tabular}

Obwohl das Liegende der Schuttmassen in vielen Aufschlüssen nicht aufgedeckt war, ist es unwahrscheinlich, daß auf den Hängen des Albtraufs auch noch Schutt aus der RißEiszeit erhalten ist, denn 1. waren die Abtragungsvorgänge während des Riß/WürmInterglazials ziemlich stark und 2. ist der Trauf der westlichen Schwäbischen Alb seit dem Riß/Würm-Interglazial um 0,5 bis $1 \mathrm{~km}$ zurückgewichen (K. BLEICH 1959).

\section{Angeführte Schriften}

Bleich, K.: Das Alter des Albtraufs. Diss. Tübingen 1959 (Ms.).

Bleich, K., \& Groscopf, P.: Periglazialbildungen am Fuße der Schwäbischen Alb bei Bad Úberkingen. - Jber. u. Mitt. oberrh. geol. Ver. N.F. 41, S. 95-102, Stuttgart 1959.

Cailleux, A.: Morphoskopische Analyse der Geschiebe und Sandkörner und ihre Bedeutung für die Paläoklimatologie. - Geol. Rdsch. 40, S. 11-19, Stuttgart 1952.

WeIPPERT, D.: Über periglaziale Fließerden und Kryoturbationen in Württemberg. - Jh. geol. L.Amt Baden-Württ. 5, Freiburg 1960. Im Druck.

Woldstedt, P.: Über die Gliederung der Würmeiszeit und die Stellung der Lösse in ihr. - Eiszeitalter u. Gegenwart 7, S. 78-86, Ohringen 1956. - - Eine neue Kurve der Würmeiszeit. Eiszeitalter u. Gegenwart 9, S. 151-154, O'hringen 1958.

Manuskr. eingeg. 3. 2. 1960.

Anschrift des Verf.: Dr. Dieter Weippert, (14a) Oberbrüden, Krs. Backnang. 